\title{
Ghislain Deslandes, Management des médias
}

Paris, Éd. La Découverte

\section{Sékouna Kéita}

\section{OpenEdition}

\section{Journals}

Édition électronique

URL : http://journals.openedition.org/questionsdecommunication/904

DOI : 10.4000/questionsdecommunication.904

ISSN : 2259-8901

\section{Éditeur}

Presses universitaires de Lorraine

\section{Édition imprimée}

Date de publication : 1 juillet 2009

Pagination : 440-443

ISBN : 978-2-86480-989-0

ISSN : $1633-5961$

\section{Référence électronique}

Sékouna Kéita, "Ghislain Deslandes, Management des médias », Questions de communication [En ligne], 15 | 2009, mis en ligne le 17 janvier 2012, consulté le 22 septembre 2020. URL : http:// journals.openedition.org/questionsdecommunication/904; DOI : https://doi.org/10.4000/ questionsdecommunication.904

Ce document a été généré automatiquement le 22 septembre 2020.

Tous droits réservés 


\section{Ghislain Deslandes, Management des médias}

Paris, Éd. La Découverte

Sékouna Kéita

\section{RÉFÉRENCE}

Ghislain Deslandes, Management des médias. Paris, Éd. La Découverte, coll. Repères, $120 \mathrm{p}$.

1 Cet ouvrage offre une analyse de l'évolution et de l'état actuel du mode de gestion des entreprises médiatiques : presse écrite, médias audiovisuels, nouveaux médias. Avec la mondialisation, deux phénomènes caractérisent la vie de ces entreprises ayant principalement en charge l'information et le divertissement. D'abord, un renforcement et une professionnalisation de leur mode de gestion à l'image des autres entreprises, avec une rationalisation des dépenses et des objectifs de performance financière et professionnelle à réaliser. Ensuite, une propension à la concentration, les médias n'échappant pas aux opérations de fusion, de rachat ou d'alliance que lancent les grands groupes industriels ou financiers, ce qui conduit à l'existence de groupes puissants, donc à une sorte de monopole de l'information que dénoncent souvent les syndicats de journalistes. Dans Le management des médias, Ghislain Deslandes apporte un éclairage appuyé d'exemples concrets sur ces réalités managériales du monde des médias. L'ouvrage comporte quatre chapitres qui décrivent différents aspects du management moderne des organes d'information et de communication, avec, selon l'auteur, une "approche qui privilégie les médias en tant qu'institutions sociales, par rapport à leur aspect purement technique » (p. 15).

2 Le premier chapitre, «La montée en puissance du management» (pp. 11-33), porte sur l'économie des médias et la place toujours plus importante qu'y prend le management au sens moderne du terme. Dans une société de l'information très marquée par la globalisation et les technologies de l'information et de la communication, les médias 
représentent un secteur économique important, fruit de plusieurs décennies de transformations dans leur mode d'organisation et de gouvernance, ainsi que dans le choix des contenus médiatiques. Face aux impératifs de résultats et de performance, indispensables en raison de la concurrence, des exigences des actionnaires et du public, le management des médias est devenu un art de faire que les patrons d'entreprises de médias intègrent et développent au rythme des mutations économiques et sociales. Avec « la hausse de la consommation des médias » (p. 12) et l'augmentation de l'offre de choix au public, l'adaptation nécessaire aux mutations fait du modèle d'organisation et de gestion des médias un facteur de succès et de solidité sur un marché où la concurrence est importante.

3 L'un des éléments caractéristiques des mutations de l'économie des médias réside dans les premières acquisitions d'entreprises médiatiques par des groupes industriels ou financiers au début des années 80. Par exemple, c'est le cas de la naissance du groupe Matra-Hachette, devenu un des plus puissants au monde après l'acquisition par Matra de Hachette et d'un groupe de presse comprenant plusieurs magasines. Pour l'auteur, l'avènement de ce grand groupe "préfigure la constitution d'ensemble capitalistiques aux moyens considérables prenant la place d'entités plus artisanales et plus indépendantes » (p. 14).En effet, qu'il s'agisse de l'Europe ou de l'Amérique du Nord, voire d'autres parties du monde, les médias sont généralement possédés par de grands groupes, traditionnellement présents dans les finances et dans l'industrie, qui les acquièrent par le biais d'opération de fusion, de rachat, de prise d'action ou d'alliance.

4 Ainsi ces géants du secteur des médias et de la communication s'assurent-ils une mainmise importante sur les médias et l'information, un secteur en plein essor. Selon des études citées par l'auteur, l'information et la fiction se portent bien, grâce à une demande constamment en hausse. Mais les acquisitions et les fusions conduisent à une concentration des médias aux mains de quelques groupes. Il n'est pas rare qu'un même groupe soit propriétaire de plusieurs chaines de télévisions et de radios, de plusieurs titres de journaux... Ce qui pose différents problèmes, notamment ceux de l'indépendance des journalistes, de la liberté d'expression, de la place de la culture. Ainsi Erik Barnouw et al. (Médias et conglomérats. Un regard sans concession sur les coulisses de l'industrie des médias aux États-Unis, trad. de l'amér. par Cécile Deniard, Paris, Éd. Liris, 2005 [1997]) dénoncent-ils la constitution de monopoles de l'information qui mettent en péril la liberté d'action des journalistes, avec les conflits d'intérêt et l'influence sur les contenus éditoriaux. Les investissements des groupes financiers et industriels dans les médias ont pour but principal de créer de la valeur ajoutée, c'est-à-dire de générer des profits financiers à travers les programmes et contenus médiatiques susceptibles de rassembler toujours la plus grande audience. Les parts de marché publicitaire en dépendent largement. Ceci pose le problème de la concurrence et de son corollairel'audimat - autour duquel les entreprises de médias s'affrontent : "Cette course pour la conquête du marché publicitaire modifie non seulement le modèle économique, mais plus encore la culture même de ces entreprises de médias: désormais on s'enthousiasme pour les études de marché et les tests d'impact, on met en place des indicateurs de la performance, on se passionne pour la rentabilité des capitaux investis» (p.15). Un des méfaits de cette «loi» de l'audimat est le retrait tout programme, même culturel, qui ne satisfait pas aux attentes en termes du nombre de personnes intéressées ou sa relégation dans des créneaux horaires moins intéressants. 
5 La nécessité de répondre aux goûts des lecteurs, auditeurs et téléspectateurs résulte notamment de l'augmentation de la consommation des produits médiatiques, ainsi que de l'offre de programmes, "car un contenu éditorial quel qu'il soit, s'il est sacré au moment de sa conception, est au final un produit qu'il faut vendre, parce qu'il coûte cher à produire » (p.19). Pour arriver à ces résultats et s'inscrire durablement dans une gouvernance performante, le manager d'entreprise médiatique développe des stratégies de diversification et d'intégration, ainsi que des alliances. À l'image d'autres entreprises commerciales, dans le secteur des médias, « la stratégie de diversification répond à l'objectif d'un groupe présent sur un ou plusieurs médias de pénétrer de nouvelles filières médiatiques » (p.2) et de réduire ainsi sa dépendance d'un seul produit ou d'une seule activité. Ghislain Deslandes donne les exemples des grandes chaines de télévisions et des groupes de presse en France et dans le monde qui sont devenus des groupes reposant sur plusieurs segments d'activités: TFI, BBC, Canal+, Financial Times, Marie-Claire... participent de cette logique qui garantit leur solidité et leur essor économique. Ici, la notion de marque revêt tout son sens avec les nombreuses déclinaisons et licences qui en découlent.

6 Le deuxième chapitre, «Le processus managérial dans les médias » (pp. 35-54), est une analyse des méthodes et principes du management appliqués aux médias. L'auteur revient sur les grandes étapes du processus managérial - planifier, organiser, diriger et contrôler - à travers des exemples de patrons d'entreprises et de leurs styles de direction. Dans ce processus et au travers de choix et de décisions stratégiques, le manager doit être capable de tenir compte des facteurs d'influence, de rationnaliser ses dépenses tout en maintenant un niveau d'investissement correct. Des mesures qui doivent permettre au groupe ou à l'entreprise de conserver toute sa compétitivité dans un marché où l'innovation et l'originalité sont des atouts majeurs. La mise en place d'une structure organisationnelle adéquate avec « des équipes talentueuses, hautement motivées et qui valorisent les marques dont elles ont la propriété » (p. 39) résulte de cette nécessité de management stratégique des entreprises de médias. Le manager est donc un acteur particulièrement important dans son rôle de dirigeant et de contrôleur. À partir de différents auteurs en ressources humaines, en management, en sociologie, Ghislain Deslandes relève quelques traits caractéristiques de personnalité : l'autorité, la passion, l'expérience, le sens de la décision, les compétences techniques, la confiance en soi, la réactivité sont quelques uns de ces traits souvent essentiels au manager qui peut se trouver face à des mutations rapides et bouleversantes. Comme dans d'autres secteurs économiques, la culture d'entreprise est de ce fait de plus en plus ancrée au niveau des managers d'entreprises médiatiques.

Dans le troisième chapitre, "Managers, médias et leadership " (pp.55-87), l'auteur centre notamment sa réflexion sur les managers eux- mêmes, leur caractère et leur parcours dans l'univers des dirigeants d'entreprises. Deux types de managers se retrouvent : d'une part, ceux qui sont en même temps propriétaires des entreprises ou groupes médiatiques dont ils ont la charge; il s'agit souvent de gens d'affaires, d'industriels ou de financiers qui acquièrent ces propriétés par rachat ou par alliance, ou qui en héritent de leur famille. D'autre part, ceux qui ne sont pas propriétaires mais qui sont nommés pour leur savoir-faire, leurs expériences et leurs compétences professionnelles. En relisant les résultats de la recherche de Monique Dagnaud et Dominique Mehl (Patrons de chaines. Les dirigeants de la télévision française au printemps 1988, Paris, Réseaux/CNET, 1990) sur les dirigeants de ces entreprises, Ghislain 
Deslandes souligne "les cinq facteurs qui«favorisent" une carrière de manager d'entreprise médiatique. Il s'agit du savoir-faire spécialisé, de la compétence plurielle, de la mobilité professionnelle, de l'appartenance à un réseau et des sympathies politiques » (p.57).

8 Pour un dirigeant de média, l'un des défis majeurs est d'allier un travail conforme à la déontologie journalistique et le maintien des objectifs de performance économique et financière. Car, tout en étant une entreprise comme toutes les autres, un média a cette spécificité d'être un acteur essentiel de la démocratie. De ce fait, s'impose à celui-ci et à ses animateurs la mission de garantir la liberté d'expression et d'assurer au public une information juste, impartiale et transparente. Entre cet impératif déontologique et les éventuels conflits d'intérêt (la nécessité de produire des résultats financièrement bons pour les actionnaires), le manager est souvent amené à faire des choix difficiles. Ce qui fait dire à l'auteur que "sur le manager des médias pèse un rôle d'arbitre entre le projet économique et le projet éditorial»(p.61). En effet, entre les objectifs de rentabilité et les choix rédactionnels, la séparation qui est un principe déontologique cardinal est souvent floue. Dans leur activité de collecte, de traitement et de diffusion de l'information, les journalistes doivent être totalement indépendants de la direction de l'entreprise. C'est un facteur de crédibilité et de transparence qui doit caractériser tout média d'information face au public. Cela étant, "le succès économique d'une publication, l'efficacité d'une organisation médiatique passent aussi par la responsabilité sociale, la liberté et la crédibilité de ses acteurs» (p. 63). C'est pourquoi Ghislain Deslandes énonce quelques éléments de culture indispensables que le manager doit posséder, tels la gestion de l'innovation, la connaissance du monde des médias et la formation technique, le souci du public et la culture du lobbying. Ces éléments sont utiles au patron d'entreprise de presse dans sa pratique du management et du leadership.

9 L'essor du management des médias comme discipline, mais aussi comme pratique professionnelle, fait l'objet du dernier chapitre: "Une discipline en mouvement » (pp. 89-111). Il est notamment question des challenges et des enjeux que les technologies numériques génèrent dans l'univers médiatique. Dans ce contexte, «la convergence des médias, le développement de nouveaux modèles économiques et des activités en ligne, et la globalisation des industries médiatiques » (p. 89) sont des défis que les managers s'acharnent à relever par des choix stratégiques qui doivent combiner originalité, performance et diversité. Le développement de la pratique de management des médias n'est pas assez suivi de travaux de recherche en France, contrairement aux États-Unis où un grand intérêt scientifique y est porté depuis plusieurs décennies. Selon l'auteur, s'il existe de nombreux travaux sur les méthodes et les principes du journalisme ainsi que sur l'économie et la sociologie des médias, il n'en est pas de même s'agissant du management stratégique des médias. Facteurs politiques, historiques et problèmes méthodologiques en sont les raisons. Mais l'émergence, en Europe et dans le monde, d'une communauté scientifique autour du management des médias pourrait signifier un début de reconnaissance de la discipline.

10 Le management des médias est donc un ouvrage qui retrace l'impact de la globalisation et des technologies numériques sur la gestion et le fonctionnement des médias dans le monde, ainsi que sur les problèmes qui en résultent. Des médias qui, pour la plupart, s'inscrivent dans des conglomérats industriels ou financiers. Mais la réflexion de l'auteur porte essentiellement sur les médias du Nord, laissant dans l'ombre la situation 
dans les pays en développement. Pour cause de fracture numérique et de déficit démocratique, les médias y connaissent un mode de gestion et de fonctionnement souvent radicalement différents. Une comparaison aurait été utile et aurait donné un éclairage plus complet sur la nature et l'essor du management des médias dans le monde actuel.

\section{AUTEURS}

\section{SÉKOUNA KÉITA}

CREM, université Paul Verlaine-Metz

sekounak@yahoo.fr 